\section{Referenzbereich, biologischer}

G. Schumann

Hannover, Deutschland

Englischer Begriff biological reference interval

Definition Festgelegter Bereich der Verteilung von Werten aus einer biologischen Referenzpopulation (ISO EN DIN 15189).

Beschreibung Bereich von Untersuchungsergebnissen einer - Messgröße aus Primärproben einer $>$ Referenzpopulation, in dem ein jeweils anzugebender Anteil der Untersuchungsergebnisse dieser Population liegt (z. B. ein zentraler $95 \%$ Bereich), wobei die Probanden an keiner Krankheit leiden, die einen Einfluss auf die untersuchte Messgröße hat. Für Anmerkungen s. Literatur.

\section{Literatur}

Medizinische Laboratorien (2014) Anforderungen an die Qualität und Kompetenz. ISO EN DIN 15 189:2014, 3, 4. Beuth-Verlag, Berlin Qualitätsmanagement in der Laboratoriumsmedizin (2001) Teil 2: Begriffe zur Qualität und Anwendung von Untersuchungsverfahren. DIN 58936-2, 6.1. Beuth-Verlag, Berlin 